\title{
Crystal Present Or Absent
}

National Cancer Institute

\section{Source}

National Cancer Institute. Crystal Present Or Absent. NCI Thesaurus. Code C74673.

The determination of the presence or absence of crystals in a sample. 